Supporting Information

\title{
A Swing Arm Location-Controllable DNA Walker for Electrochemiluminescence Biosensing
}

\author{
Ni Liao ${ }^{a, b}$, Mei-Chen Pana, Li Wanga, Fan Yang ${ }^{a}$, Ruo Yuana, Ying Zhuo ${ }^{a, *}$ \\ a Key Laboratory of Luminescence Analysis and Molecular Sensing (Southwest \\ University), Ministry of Education, College of Chemistry and Chemical Engineering, \\ Southwest University, Chongqing 400715, PR China \\ b College of Biological and Chemical Engineering, Panzhihua University, Panzhihua \\ 617000, China
}

\footnotetext{
* Corresponding authors at: Tel.: +86 23 68253172, fax: +86 2368253172 .

E-mail addresses: yingzhuo@swu.edu.cn (Y. Zhuo)
} 


\section{Table of contents}

1.1 Reagents and Materials.

1.2 Apparatus S4

1.3 Preparation of the Pure Pe MCs and TAPE MCs. S5

1.4 Preparation of Ag Nanoparticles (Ag NPs). S5

1.5 The Microstructure and Zeta Potential Characterization of Various Nanomaterials. S6

1.6 The Images of Pe and TAPE with Different States and the ECL Spectrum of $\mathrm{S}_{2} \mathrm{O}_{8}{ }^{2-}$ S8

1.7 ECL and Electrochemical Impedance Spectroscopy (EIS) Characterizations of the Proposed Biosensor. S8

1.8 Optimization of the Reaction Conditions. $\mathrm{S} 10$

1.9 The Relative ECL Efficiency of TAPE-Pe MCs S11

References $\mathrm{S} 12$ 


\subsection{Reagents and Materials.}

Perylene $(\mathrm{Pe})$ was received from Lian Gang Dyestuff Chemical Industry Co. Ltd. (Liaoning, China). Tetrahydrofuran (THF) was obtained from J\&K Scientific Ltd. (Beijing, China). Tetrakis (4-aminophenyl) ethane (TAPE) was provided by Huawei Ruike Chemical Co. Ltd. (Beijing, China). Hexadecyl trimethyl ammonium bromide (CTAB) was supplied by Kelong Chemical Reagent Plant (Chengdu, China). Poly (diallyldimethylammonium chloride) (PDDA, 50\% wt in water, molecular weight 20000), was purchased from Aldrich. The phosphate buffer saline (PBS, 0.1 $\mathrm{M}, \mathrm{pH}$ 7.4) consist of $0.1 \mathrm{M} \mathrm{KH}_{2} \mathrm{PO}_{4}, 0.1 \mathrm{M} \mathrm{Na}_{2} \mathrm{HPO}_{4}$ and $0.1 \mathrm{M} \mathrm{KCl}$. The $\mathrm{S}_{2} \mathrm{O}_{8}{ }^{2-}$ solution (10 mM, pH 7.4) was prepared by dissolving a certain amount of solid $\mathrm{K}_{2} \mathrm{~S}_{2} \mathrm{O}_{8}$ in $0.1 \mathrm{M}$ PBS. The DNA oligonucleotides, tris-(2-carboxyethyl)-phosphine hydrochloride (TCEP) and exonuclease III (Exo III) was obtained from Sangon 
Biotech Co., Ltd. (Shanghai, China). In addition, the other involved solutions in this work were prepared using deionized water, which derived from Milli-Q water purification system with an electric resistance of $18.2 \mathrm{M} \Omega$.

The sequences of sequence information for the nucleic acids used in this study were shown in Table S1:

Table S1. Sequence Information for the Nucleic Acids Used in This Study.

\begin{tabular}{cl}
\hline \hline Name & Sequence* $^{*} \mathbf{( 5} \boldsymbol{- 3}^{\mathbf{3}} \mathbf{)}$ \\
\hline \hline let-7a & UGA GGU AGU AGG UUG UAU AGU U \\
locking & TGAATAACTATACAACCTACTACCTCA \\
strands & \\
swing arm & \\
strands & TCGGAACGGCTTATCTGTTACT ${ }_{25}$ AGGTTGTATAGTTATTCATTTTTTT \\
S1 & GTAACAGATAGGCCAATGCTCACGGCGGAGATTACGACGAGACACA \\
& TGGGAACCGAACAATGTTTTTTTGAATAACTATACAA-Fc \\
S2 & AGCATTGGCCATGAAACTCCCAGCGAGCAGCAGGAACGTCCGAATG \\
& ATATAAACTCCGCCGTGTTTTTTTGAATAACTATACAA-Fc
\end{tabular}


Furthermore, various kinds of buffers applied in the research are listed as follows:

(1) $1 \times$ Tris-EDTA buffer (TE buffer, $10 \mathrm{mM}$ Tris- $\mathrm{HCl}, 1.0 \mathrm{mM}$ ethylene diamine tetraacetic acid (EDTA), $\mathrm{pH}$ 8.0) was used for the dissolution and storage of template and walker.

(2) DNA hybridization buffer (containing $10 \mathrm{mM}$ Tris-HCl, $1.0 \mathrm{mM}$ EDTA, and 1.0 $\mathrm{M} \mathrm{NaCl}, \mathrm{pH}$ 7.0) was used as buffer solution for DNA hybridization;

(3) Tris-Magnesium sulfate buffer (TM buffer, $10 \mathrm{mM}$ Tris- $\mathrm{HCl}$ and $50 \mathrm{mM}$ $\mathrm{MgCl}_{2}, \mathrm{pH}$ 8.0) was used to obtain the DNA tetrahedron nanostructures (DTNs) for the self-assembly of four single strands of DNA (S1, S2, S3 and S4).

\subsection{Apparatus}

The ECL and electrochemical measurements were performed on a MPI-A multifunctional analyzer (Xi'An Remax Electronic Science \& Technology Co. Ltd., Xi'An, China) and CHI 660E electrochemistry workstation (Shanghai $\mathrm{CH}$ Instruments, China) with the conventional three-electrode system (a platinum wire as counter electrode, a modified glass carbon electrode (GCE, $\Phi=4 \mathrm{~mm}$ ) as working electrode, and an $\mathrm{Ag} / \mathrm{AgCl}$ (sat. $\mathrm{KCl}$ ) as reference electrode). Fluorescence spectra were characterized by FL-5700 spectrophotometer (Hitachi, Tokyo, Japan). The 
surface morphologies of different nanomaterials were characterized by S-4800 scanning electron microscopy (SEM, Hitachi, Tokyo, Japan). ECL emission spectra were analyzed by ECL spectrum system built by our laboratory, which mainly included the electrochemical workstation (Vertex, Ivium Technologies Co., Ltd., Netherlands), the spectrometer (Kymera 193i, Andor, U.S.A) and Multi-spectral EMCCD (Newton, Andor, U.S.A) combined with a Newton EMCCD spectroscopy detector (Andor Co., Tokyo, Japan).

\subsection{Preparation of the Pure Pe MCs and TAPE MCs.}

The pure Pe MCs were prepared as following: First, $2.5 \mathrm{mg}$ Pe powder was dissolved in $2.5 \mathrm{~mL}$ of THF with vigorous stirring to prepare a homogeneous $\mathrm{Pe}$ solution, which was rapidly added into $10 \mathrm{~mL}$ CTAB aqueous solution $(15 \mathrm{mmol} / \mathrm{L})$ under rapid agitation for $20 \mathrm{~min}$. Then the Pe MCs were collected through centrifugation and washed several times using deionized water to remove residual THF and CTAB. Finally, the Pe MCs were redispersed in $10 \mathrm{~mL}$ of deionized water for subsequent use. The TAPE MCs were prepared according to the above route by replacing Pe with TAPE.

\subsection{Preparation of Ag Nanoparticles (Ag NPs).}

The synthesis of the Ag nanoparticles (Ag NPs) was according to the previous method ${ }^{1}$. In brief, the $\mathrm{AgNO}_{3}$ aqueous solution $(0.5 \mathrm{~mL}, 1 \mathrm{~mol} / \mathrm{L})$ and the poly(vinyl pyrrolidone) (PVP) aqueous solution $(2.5 \mathrm{~mL}, 1 \mathrm{~mol} / \mathrm{L})$ were added to $25 \mathrm{~mL}$ of poly(ethylene glycol) 600 (PEG 600) in a flask under stirring. In this, the PVP as surfactant and PEG 600 as reducing agent. The flask was transferred to an oil bath and 
heated at $100{ }^{\circ} \mathrm{C}$ for about $8 \mathrm{~h}$. The synthesized Ag NPs were collected by centrifugation and washing with ethanol three times.

\subsection{The Microstructure and Zeta Potential Characterization of Various Nanomaterials.}

The microstructure of the TAPE-Pe MCs was characterized by SEM. The SEM image of TAPE-Pe MCs exhibited a uniform blocklike structure with $\sim 400 \mathrm{~nm}$ in length and in width, as showed in Figure S1A. In addition, zeta potential is a common method to verify the surface charges of nanomaterials. The zeta potentials of TAPE-Pe MCs and Ag NPs were measured at $\mathrm{pH}=7.4$, respectively, and the related data was displayed in Fig. S1B. Zeta potential value of TAPE-Pe MCs and Ag NPs were about $-8.39 \mathrm{mV}$ and $-8.0 \mathrm{mV}$, respectively. These indicating that TAPE-Pe MCs and Ag NPs were negatively charged. Therefore, the PDDA was introduced to obtain the positive charged TAPE-Pe MCs and the Ag NPs adhered to the TAPE-Pe MCs surface via electrostatic adsorption. The microstructure of the Ag NPs was characterized by transmission electron microscopy (TEM), the TEM images of the Ag NPs showed an orbicular structure with the diameters of about $60 \sim 80 \mathrm{~nm}$, as shown in Figure S1C. And the enlarged TEM image of Ag NPs was shown in Figure S1D. 

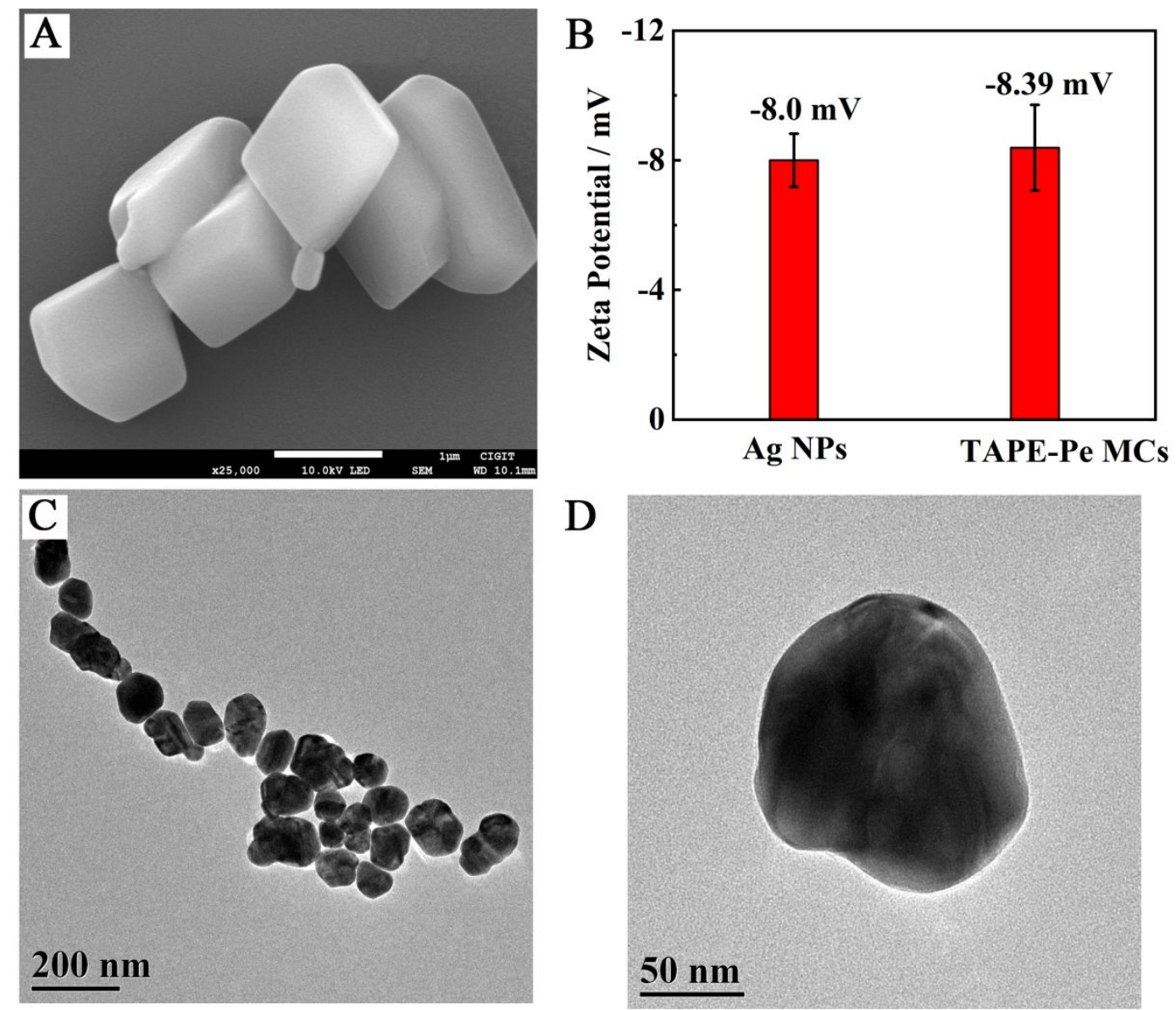

Figure S1. The SEM image of TAPE-Pe MCs (A). The zeta potential of TAPE-Pe MCs and Ag NPs (B). The TEM images of Ag NPs (C) and the enlarged TEM image of Ag NPs (D). 
1.6 The Images of Pe and TAPE with Different States and the ECL Spectrum of $\mathrm{S}_{2} \mathrm{O}_{8}{ }^{2-}$.

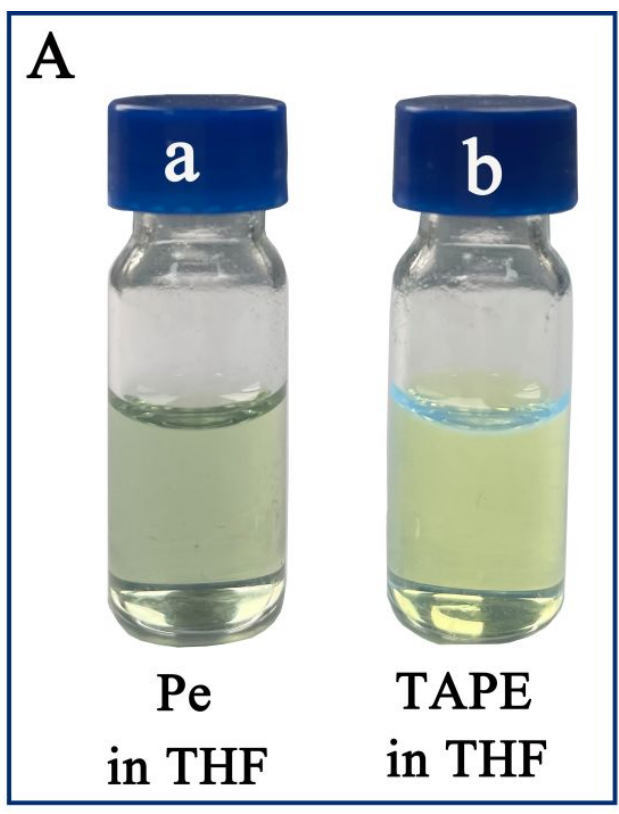

C

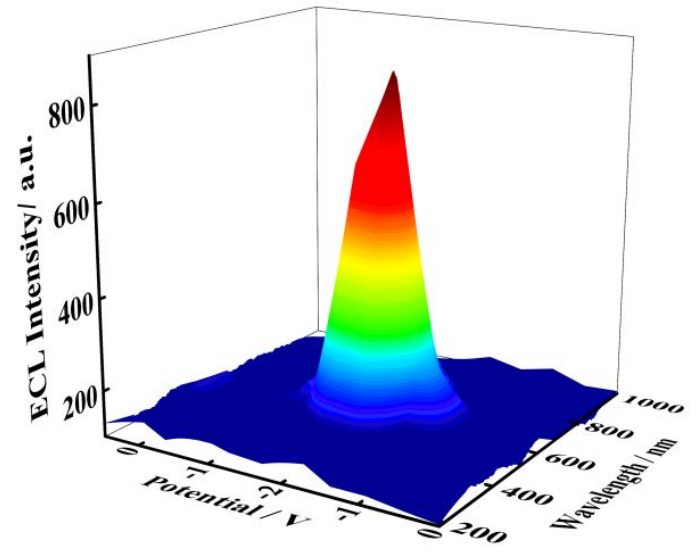

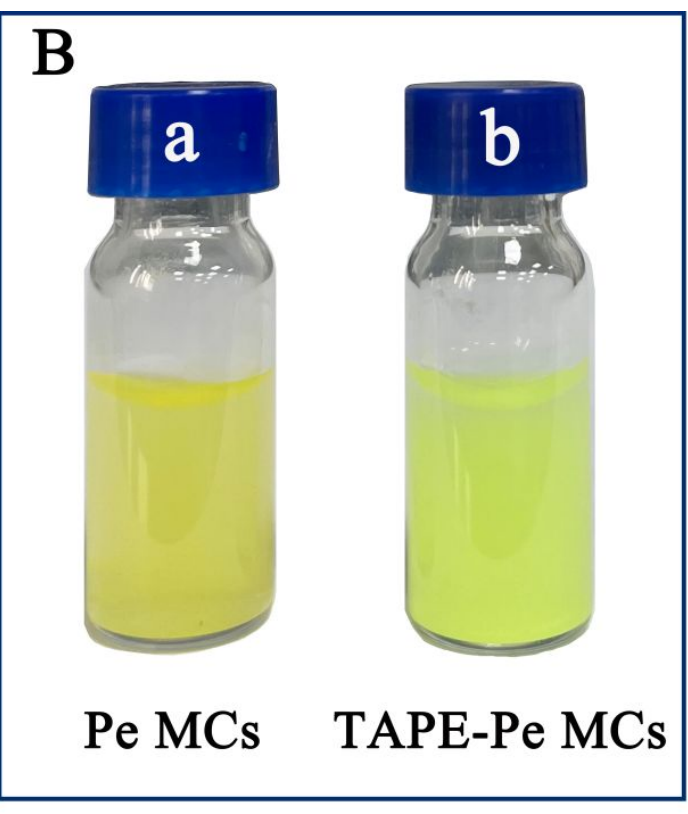

D

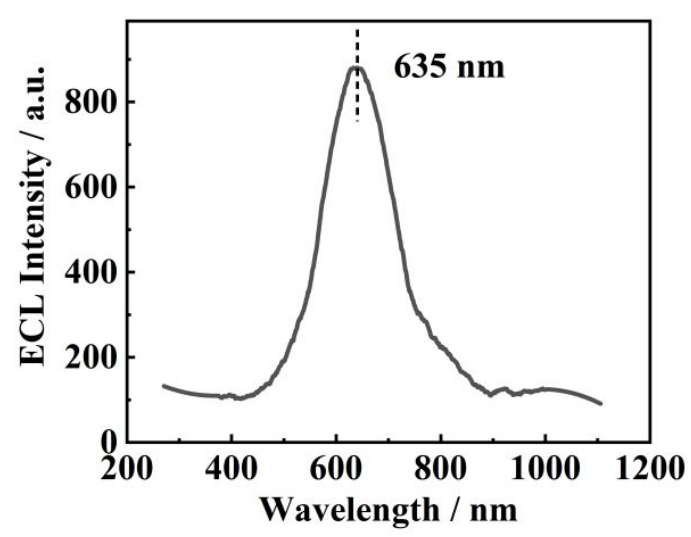

Figure S2. The images of Pe (a) and TAPE (b) dissolved in THF under the natural light (A). The images of Pe MCs (a) and TAPE-Pe MCs (b) in deionized water under the natural light (B). The ECL-potential-wavelength of the GCE in $\mathrm{S}_{2} \mathrm{O}_{8}{ }^{2-}$ solution via a 3D surface image (C) and the 2D ECL spectrum of the GCE in $\mathrm{S}_{2} \mathrm{O}_{8}{ }^{2-}$ solution (D).

\subsection{ECL and Electrochemical Impedance Spectroscopy (EIS) Characterizations}

of the Proposed Biosensor.

To characterize the stepwise fabrication process of the biosensor, the ECL and 
EIS measurements of different assembled electrodes were conducted in PBS (pH 7.4) with $\mathrm{S}_{2} \mathrm{O}_{8}{ }^{2-}$ as the coreactant (Figure $\mathrm{S} 3 \mathrm{~A}$ ) and $5.0 \mathrm{mM}\left[\mathrm{Fe}(\mathrm{CN})_{6}\right]^{3-/ 4-}$ as redox probes (Figure S3B) under the scan potential range from -2 to $0 \mathrm{~V}$, respectively. As presented in Figure S3A, the bare GCE (curve $a$, Figure S3A) showed no distinct ECL emission in PBS owing to the lack of ECL luminophores. When the TAPE-Pe MCs dripped the GCE (TAPE-Pe MCs/GCE), a remarkable ECL signal was obtained due to the presence of TAPE-Pe MCs acted as ECL luminophore (curve $b$, Figure S3A). When the Ag NPs was assembled onto the modified electrode, a slight augment of the ECL signal was presented (curve $c$, Figure S3A). Subsequently, the ECL intensity declined promptly as the modified electrode incubated with location-controllable DNA walker solution (curve $d$, Figure S3A), attributing to the quenching effect of Fc from location-controllable DNA walker. Subsequently, the ECL signal further decreased because the HT could obstruct the electron transfer (curve $e$, Figure S3A). However, the ECL intensity (curve $f$, Figure $\mathrm{S} 3 \mathrm{~A}$ ) recovered partially after the reaction solution containing $10^{-10} \mathrm{M}$ let-7a was incubated on the modified GCE because the swing arm strand was activated to hybridize with track strands, resulting in the release of the Fc with the help of Exo III.

In addition, the EIS is an effective method for studying the interface properties of modified electrodes and the electron-transfer resistance at the electrode surface. Figure S3B exhibited the EIS plots of the fabrication process at each step. The bare GCE showed a small semicircle (curve $a$, Figure S3B) due to the low electron-transfer resistance $\left(\mathrm{R}_{\mathrm{et}}\right)$ value in PBS containing $5.0 \mathrm{mmol} / \mathrm{L} \mathrm{Fe}(\mathrm{CN})_{6}^{3-/ 4-}$. The $\mathrm{R}_{\mathrm{et}}$ value 
increased slightly after the TAPE-Pe MCs was decorated on the electrode (curve $b$, Figure S3B), which could be assigned to the formation of TAPE-Pe MCs layer on the electrode surface. When the modified electrode was incubated with the Ag NPs, the $\mathrm{R}_{\mathrm{et}}$ value decreased as Ag NPs accelerated the electron transfer (curve $c$, Figure S3B). When the location-controllable DNA walker was introduced on the electrode surface (curve $d$, Figure $\mathrm{S} 3 \mathrm{~B}$ ) and then blocking with $\mathrm{HT}$ (curve $e$, Figure $\mathrm{S} 3 \mathrm{~B}$ ), the $\mathrm{R}_{\mathrm{et}}$ response increased in succession because they could impede the electron transfer. The $R_{\text {et }}$ response decreased after the incubation of the reaction solution containing $10^{-10} \mathrm{M}$ let-7a (curve $f$, Figure S3B) as the Fc modified track strands left from the electrode surface with the help of Exo III.
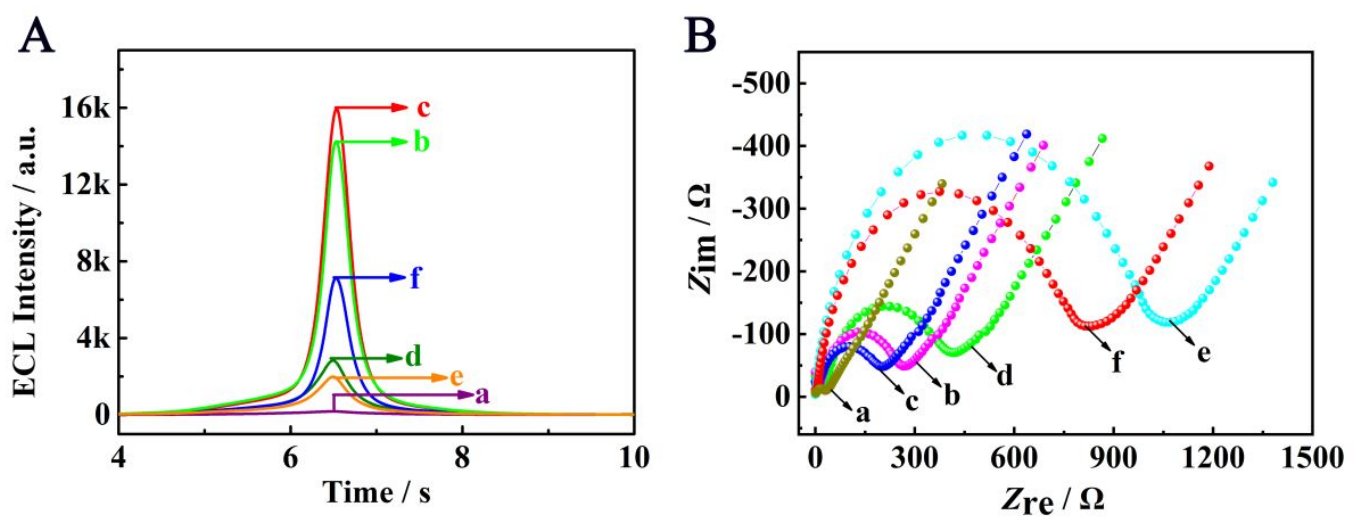

Figure S3. Typical ECL (A) and EIS (B) profiles recorded on different modified electrodes in 2 $\mathrm{mL}$ PBS (pH 7.4) containing $10 \mathrm{mM} \mathrm{S}_{2} \mathrm{O}_{8}{ }^{2-}$ and $5.0 \mathrm{mM}\left[\mathrm{Fe}(\mathrm{CN})_{6}\right]^{3-4-4}$, respectively. (a) GCE, (b) TAPE-Pe MCs/GCE, (c) Ag NPs/TAPE-Pe MCs/GCE, (d) DNA walker/Ag NPs/TAPE-Pe MCs/GCE, (e) HT/DNA walker/Ag NPs/TAPE-Pe MCs/GCE, (f) the reaction solution/HT/DNA walker/Ag NPs/TAPE-Pe MCs/GCE.

\subsection{Optimization of the Reaction Conditions.}

In order to achieve the optimal sensing performance, the main conditions 
including the incubation time of DNA walker solution and reaction solution on the modified electrode were studied, respectively. The results were depicted in Figure S4. The ECL intensity decreased along with the increment of the incubation time and tended to be stable after $6 \mathrm{~h}$ when the DNA walker solution was incubated on the modified electrode at $25{ }^{\circ} \mathrm{C}$ (Figure S4A). It could be assigned to the Fc labeled track strands could quench ECL response of TAPE-Pe MCs. Besides, the incubation time of reaction solution (containing $10 \times$ Exo $\mathrm{III}$ buffer, $500 \mathrm{U} / \mu \mathrm{L}$ Exo $\mathrm{II}$ and the target let-7a) on the modified electrode was also optimized as shown in the Figure S4B, the ECL signal increased rapidly with the increasement of the reaction time and stabilized eventually when the incubation time was over $2 \mathrm{~h}$, thus the $2 \mathrm{~h}$ was selected as the optimal incubation time of reaction solution on the modified electrode in the following experiments.
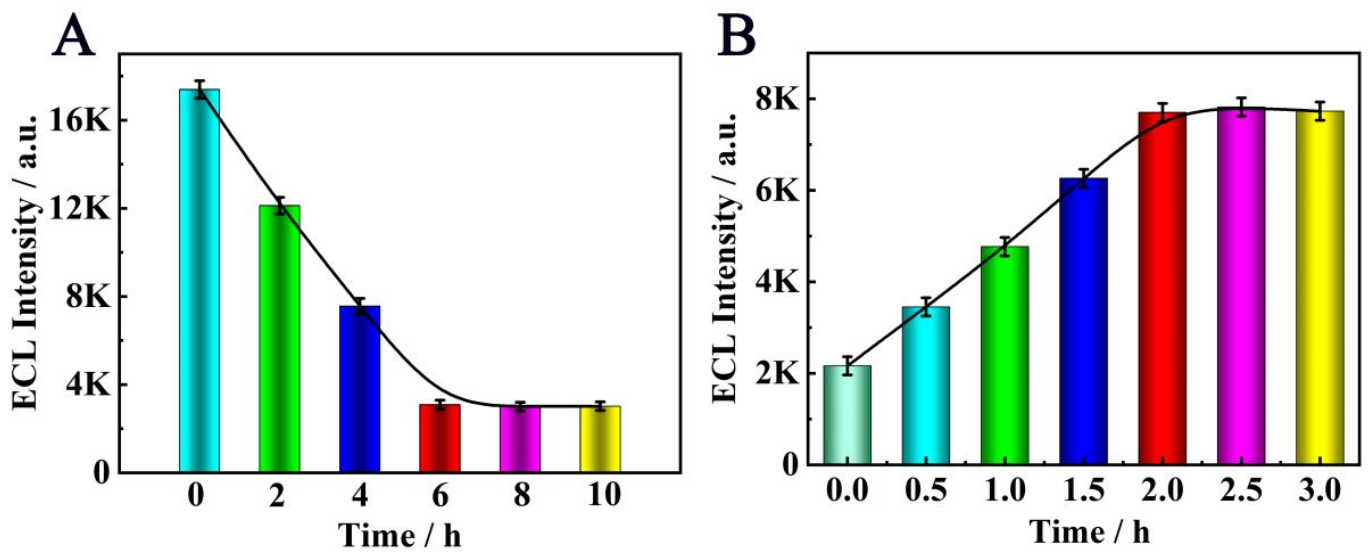

Figure S4. The incubation time of DNA walker solution on the electrode (A) and the reaction solution on the modified electrode (B).

\subsection{The Relative ECL Efficiency of TAPE-Pe MCs.}

The relative ECL efficiency of TAPE-Pe MCs was calculated by comparing the ratio of the integrated ECL intensity of the TAPE-Pe MCs in reference to that of Pe 
MCs as the following equation:

$$
Q=\frac{\Phi_{x}}{\Phi_{0}}=\left(\frac{\int_{0}^{t} I d t}{\int_{0}^{t} i d t}\right)_{x} /\left(\frac{\int_{0}^{t} I d t}{\int_{0}^{t} i d t}\right)_{0}
$$

Here, $\Phi_{x}$ is the ECL quantum efficiency of TAPE-Pe MCs, $\Phi_{0}$ is the ECL quantum efficiency of Pe MCs. Where " $P$ " is ECL intensity, " $i$ " is current value.

\section{References}

1 Wei, H.; Coronado, A. R.; Nordlander, P.; Aizpurua, J.; Xu H. X. Multipolar plasmon resonances in individual Ag nanorice. ACS Nano, 2010, 4, 2649-2654. 